\title{
Spatial Super-Compression of the Continuous Media in High-Frequency Fields
}

\author{
Kholmurad Khasanov \\ Gas and Wave Dynamics Department, M. V. Lomonosov Moscow State University, Moscow, Russia
}

Email address:

kholkh@bk.ru

\section{To cite this article:}

Kholmurad Khasanov. Spatial Super-Compression of the Continuous Media in High-Frequency Fields. American Journal of Modern Physics. Vol. 4, No. 6, 2015, pp. 281-286. doi: 10.11648/j.ajmp.20150406.14

\begin{abstract}
Firstly there is established unknown early the spatial super-compression of continuous media at the open air atmosphere which is caused by self-organizing criticality and accompanied with electromagnetic radiation of high energy. Also during the spatial super-compression the glowing of the continuous media with high extent of ionization is observed. The presented experiments are a total analysis of the previously works which were discussed in the articles on this theme. The experiments show that energy of HF sound transforms into the energy HF electromagnetic field. This process leads to the spatial super-compression of gas. This phenomenon was researched using the dynamic emitter as during outflowing of gas from it as without it. The experiments were carried out as during supplying of HF electromagnetic field, as without it. But at the both cases the spatial super-compression and glowing of the continuous media were observed. Early at our previously works we discussed super-compression of submerged supersonic jet and its high energy emitting which was registered by standard spectrographs. Characteristics of obtained spectrums attest about the emitting at violet, ultraviolet areas and also Xray area.
\end{abstract}

Keywords: Spatial Super-Compression, High-Frequency Fields, Continuous Media, Supersonic Jet, Dynamic Emitter, Self-Organizing Criticality, High Energy Radiation

\section{Introduction}

We early have discussed phenomena of supercompressibility in supersonic spiral-twisted jets [1], [2], emission of high energy during super-compressibility of supersonic jets. In that works we have visualized the first super-compressibility and associated it phenomena in mentioned spiral-twisted gas jets during its flowing from dynamic emitter. In this work we present the other experiments which are the logical consequence of the previous researching and new experiment results according to HF super-compression of gas and the formation of high density of gas in the submerged supersonic jets and HF electric fields without using of the jet [2]. We established that unhomogeneity gas density distribution leads to selforganizing electromagnetic field criticality which creates the spatial super-compression. There are not the information about laboratory methods of researching of HF supercompression of continuous media and the formation of high gas density. The data presented in this work gives us a possibility to study these phenomena under station laboratory conditions. We think that the discovered phenomena will be interest for wide audience.

Analogical to the spatial super-compression of continuous media we can compare pinch-effect. Many scientists have researched this effect [3 - 9]. At these works high current density is used at areas of which strong magnetic fields occur and we have another physical conditions in which we demonstrate the spatial super-compression of continuous media which is different from pinch-effect in principle.

In this work due to our researchings and explanation of HF super-compression of gas and the formation of high density of continuous media at the open space and unique configuration of nozzle with central cone we can study this interesting phenomenon under lab stationary conditions.

\section{Experimental Part}

We think it needs present the detail configuration parameters of the nozzle which let us to observe the phenomenon of HF spatial super-compression and results of our previously experiments. Design of the dynamic emitter in 
Fig.1 allows a lengthwise movement of the conic central body 1 inside the truncated cone 2 for a different disposition of its top relative to a cut of the dynamic emitter. At the previous works we described very detail a configuration of the dynamic emitter and calculations which are related with the definition of the parameters of the researching matter such as the energy, velocity, enthalpy and mass. More detail information about this you can see at early published works [10], [11], [1]. Here we just show a picture of the construction of the dynamic emitter at Fig. 1.

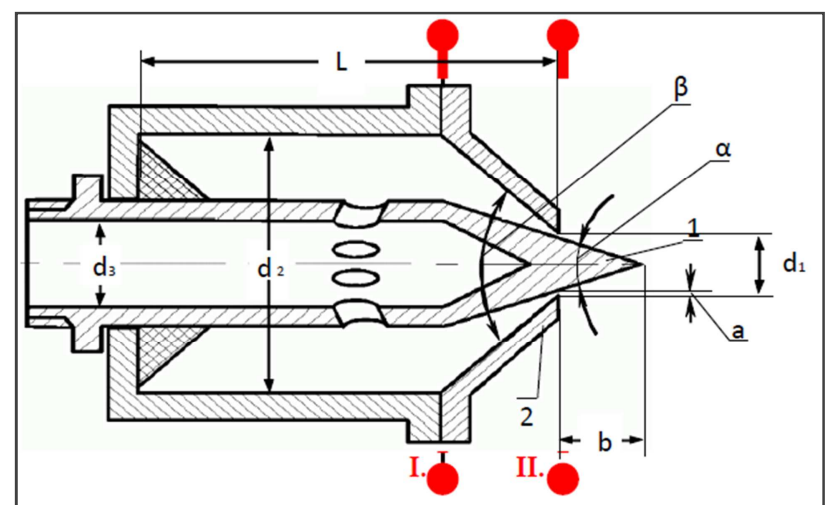

Fig. 1. Design of the dynamic emitter with central cone. Here: 1 is the central cone with diameter $d_{3}=25 \mathrm{~mm}$ and the angel $\alpha=30 ; 2$ is the truncated cone of the pre-chamber with diameter $d_{2}=60 \mathrm{~mm}$ and the angel $\beta=60 ; d_{1}$ is a diameter of outlet of the nozzle; the annular gap a $=0.3-0.6$ $\mathrm{mm}$; the external part of the internal cone $b=3-6 \mathrm{~mm}$; a length of the prechamber $L=150 \mathrm{~mm}$. The dimensions are given in [6].

\subsection{The Laser Visualization of the HF Spatial Super- Compression and the Formation of High Gas Densities}

Early there was written about this construction of this nozzle and structure occurring during the flowing of gas through the dynamic emitter at works [10], [11]. Spherical symmetrical formations of the high gas densities were observed during the outflowing of air from this construction which are presented on the Fig. 2 [12]. White arrows show to the dynamics of the gas spatial super-compression at the submerged supersonic jet. Many experiments carried out at the further works of researching of the molecules' excited states and definition of energy density at cubic volumes. Then we didn't have enough data which can explain the mechanism of the formation of these spherical symmetrical formations which are occurred due to the transformation of HF sound field into HF electromagnetic field. Then we told only about super-compressibility of supersonic jets [1]. The further experiments led us to the energy densities of these formations are more greatly than the energy densities of the shock waves which appear in supersonic jets. There is shown the laser visualization of the high gas densities at the Fig. 2. The white arrow shows on one of the formations. In this experiment the dynamic emitter with the configuration parameters presented at Fig.1 was used. During supplying air from compressor to pre-chamber under pressure $6 * 10^{5} \mathrm{~Pa}$, $293 \mathrm{~K}$ air temperature and the air humidity is $98 \%$, the jet temperature is $285 \mathrm{~K}$, the frequency of HF sound field is from $25 \mathrm{kHz}$ to $1 \mathrm{MHz}$. In the submerged supersonic jet the structures presented at Fig. 2 occur. Such structures were discussed at the works $[1,13]$. During superhighspeed video we can observe how semispherical formations merge in whole spherical symmetrical formation.
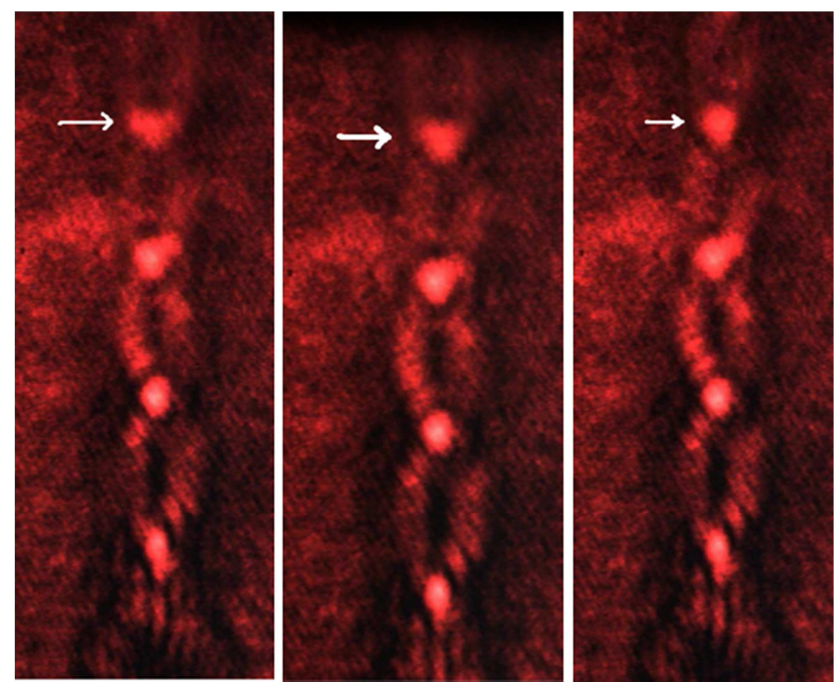

Fig. 2. Dynamic emitter with large annular slit, $b=5 \mathrm{~mm}$, nozzle diameter $d$ $=5 \mathrm{~mm}$, pressure $6 * 10^{\wedge} 5 \mathrm{~Pa}$. Vertical position of flow direction. White arrow shows one of the formations of high gas densities in the structure of supersonic jet.

\subsection{The Visualization of Spatial Super-Compression of Continuous Media by Supersonic Jet}

\subsubsection{The Stationary Mode}

These experiments were carried out at the open air, the visualization of the flows was realized using Toepler device. The Fig. 3 shows super-sonic gas air jet which is outflowing from the dynamic emitter to submerged space and its direction is by gravity vector. The air temperature is $293 \mathrm{~K}$ and the air humidity is $98 \%$. At the Fig. 4 we observe the situation when flame of the wax candle from below from dynamic emitter at the distance of $20 \mathrm{~cm}$ without the jet. And the Fig. 5 and Fig. 6 present us transform of HF sound field to $\mathrm{HF}$ electromagnetic field where the spatial supercompression of continuous media occurs and we observe spherical symmetrical formations of high gas densities. The sound frequency of field was from $25 \mathrm{kHz}$ to $1 \mathrm{MHz}$. And here our calculations showed that density of energy in these formations mounts to $3 * 10^{5} \mathrm{~J} / \mathrm{sm}^{3}$. When the jet begins to outflow from dynamic emitter we observe the spatial supercompression and the formation of high gas densities at the open air. At the Fig.5 white arrow show on the area of the spatial super-compression. At the Fig.6 increasing the pressure in pre-chamber from $6 * 10^{5} \mathrm{~Pa}$ to $8 * 10^{5} \mathrm{~Pa}$ we can clearly observe the formations not only at the place where thermal jet of the candle are transformed in the formation of gas high density but after the cut of the emitter. It is the result of influence of the jet in which the particles are under high presser and high accelerations [13]. 


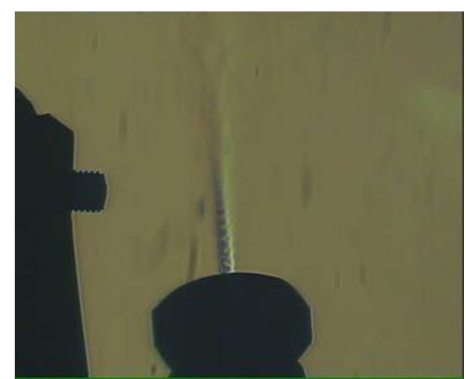

Fig. 3. Demonstration of the formation spherically symmetrical formations in the supersonic jet. Pre-chamber pressure is $6 * 10^{5} \mathrm{~Pa}$.

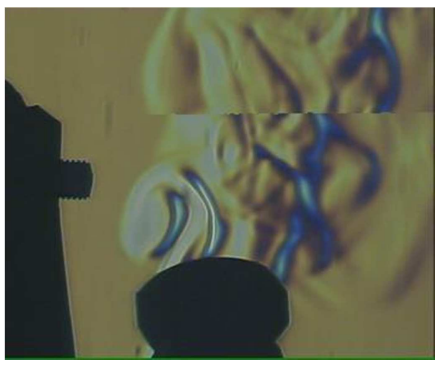

Fig. 4. This figure show us along the thermal flow from flame of candle without jet.

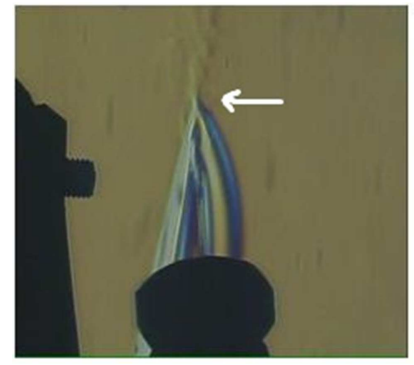

Fig. 5. Demonstration of the spatial super-compression of thermal flow from flame of candle. It happens in a trice during $3 * 10^{-3}$ s. The pressure is $6^{*} 10^{5}$ $\mathrm{Pa}$.

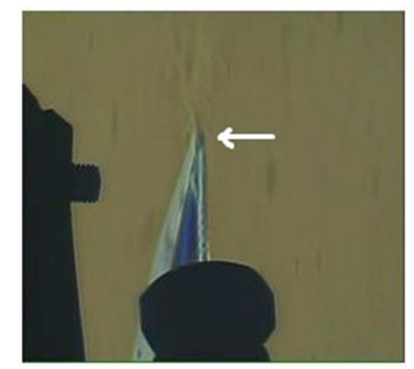

Fig. 6. The formations not only at the place where thermal jet of the candle are transformed in high gas density formations but after the cut of the emitter. The pressure is $8 * 10^{5} \mathrm{~Pa}$.

\subsubsection{The Dynamic Mode}

We present the experiment in which dynamic emitter is oriented on horizon by $45^{\circ}$. The nozzle and flame of the wax candle are on the experimental booth which is on rail. Video is made under dynamic conditions and in automatic mode. All physical conditions are the same as at the first experiment. During the outflowing of supersonic air jet at submerged space in interaction with thermal flow of candle flame we clearly observe the spatial super-compression and the formation of spherical symmetric compressed areas of gas supersonic jet.

These pictures demonstrate changes of structures and its behavior during removing of the dynamic emitter, which is located on the mobile rail. The Fig. 7 show us situation without the flowing of jet and there is just burning of wax candle flame which is by $20 \mathrm{~cm}$ from the nozzle central cone. The Fig. 8 shows us the spatial super-compression of the continuous media and the formation of spherical symmetrical high gas and thermal flow densities at the jet. Although thermal flow from wax candle is far from the nozzle cut the spatial supercompression of the flow is observed as ball-shaped formations. At the previous works we said that during this process the continuous media can condense [14]. Then while the nozzle goes away from the candle we see twisted spherical symmetrical formations (Fig. 9) [15]. Further we decrease the pre-chamber pressure from $8 * 10^{5} \mathrm{~Pa}$ to $2 * 10^{5} \mathrm{~Pa}$ and bring thermal wax candle flow closer to the nozzle cut. And we see the changes of structure of the formations. At the Fig. 10, Fig. 11, Fig. 12 we see the formations of high gas densities although the decreasing of the pressure. The changing of distance between thermal flow and jet and pre-chamber pressure lead to unessential changing of the formation of high gas densities. The jet is so strong that even being on the distance from the flame these formations is taking place.

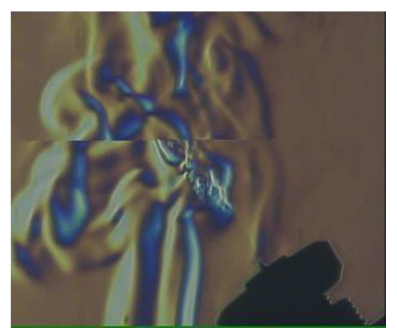

Fig. 7. The demonstration of thermal flow which is bellow about $20 \mathrm{~cm}$ from the nozzle.

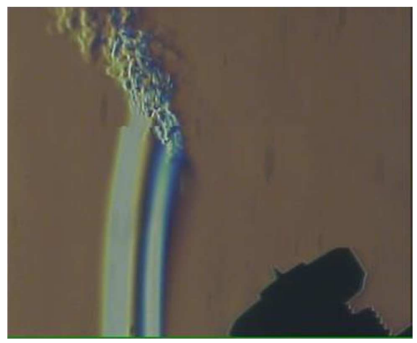

Fig. 8. The interaction the jet with thermal flow. The pressure is $8 * 10^{5} \mathrm{~Pa}$.

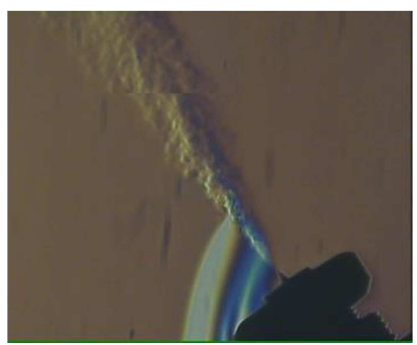

Fig. 9. The distance between the nozzle and thermal flow is decreased. The pressure is $8 * 10^{5} \mathrm{~Pa}$. 


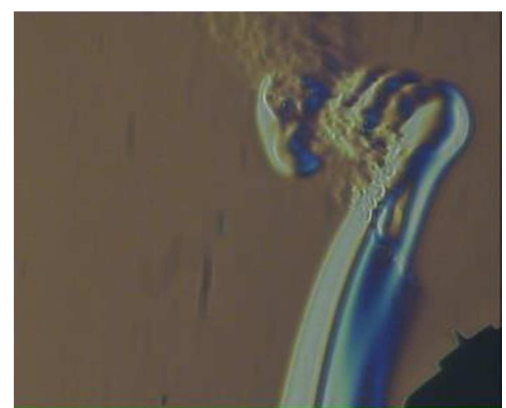

Fig. 10. The demonstration of the annual structure under low pressure $2 * 10^{5}$ $\mathrm{Pa}$.

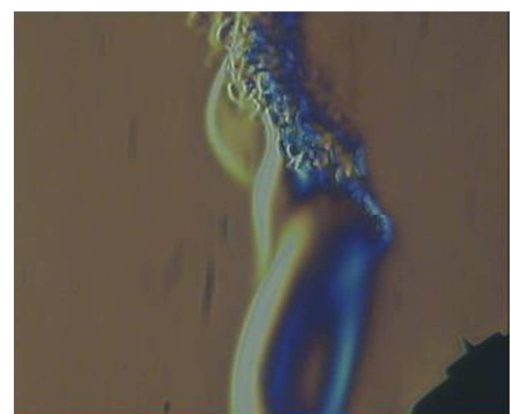

Fig. 11. The dynamic of the process of the twisting of the jet and the formation of high gas densities. The pressure is $6 * 10^{5} \mathrm{~Pa}$.

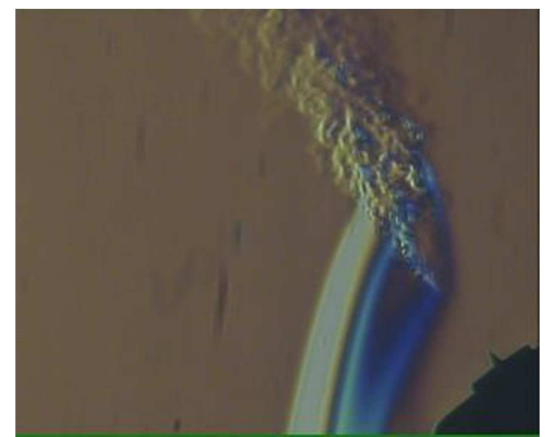

Fig. 12. The demonstration of the spherical symmetrical formations with vortex structure. The pressure is $4 * 10^{5} \mathrm{~Pa}$.

\subsection{The Formation of the Spatial Super-Compression of Continuous Media During HF Electromagnetic Field}

The formation of the spatial super-compression of continuous media was recorded by a camera and below we present the photographs made from a superhighspeed video, which was made under the conditions without a pressure and without a jet. (Fig. 13, Fig. 14). The cone is a metal - bronze - and the other parts of the dynamic emitter are a dielectric. The megahertz HF field by $1.5 \mathrm{MHz}$ is supplied to the cone and we can see at the Fig. 13, Fig. 14 the spherical symmetrical formations of high density air media. We observe how the spatial super-compression and the emitting of light energy high density occur. All these phenomena are caused by strong irregular distribution of continuous media density which is result of the strong instability. In these cases at the submerged space the self-organizing criticality is occurred and it leads to the phenomenon of the continuous media spatial super-compression.
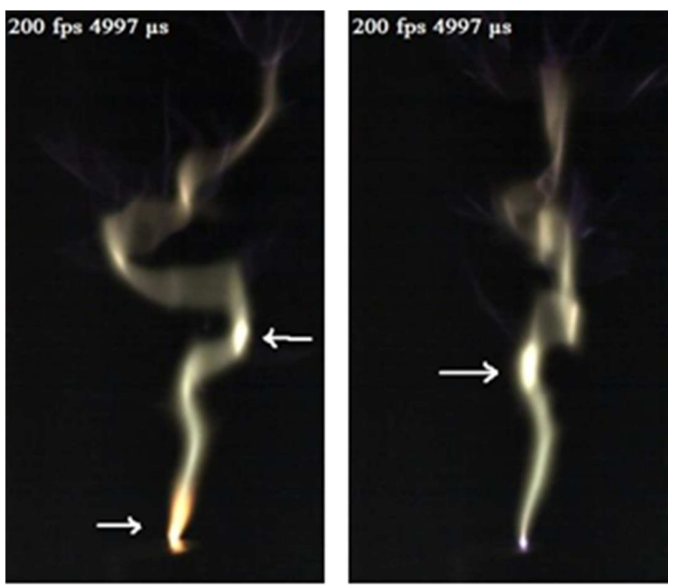

Fig. 13. The formation of the high gas densities at the metal cone without pressure and in the megahertz HF field. We can observe the dynamics of these formations step by step.
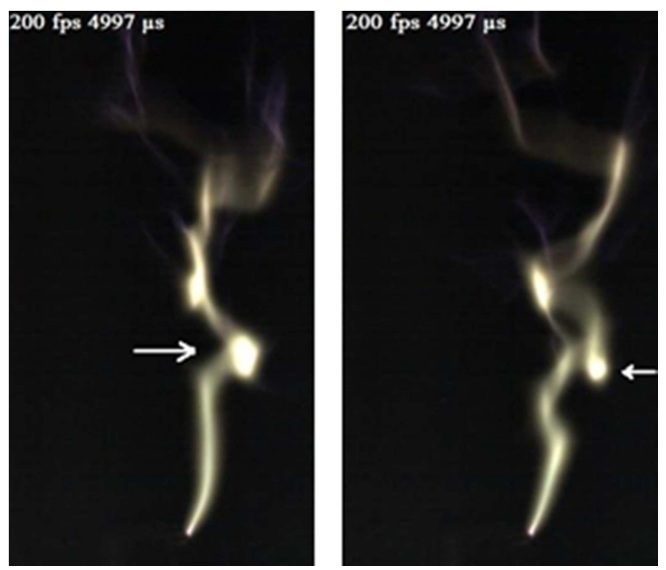

Fig. 14. We can observe the dynamics of this process on these photos. As a result we have got series of the fireballs running out from the nozzle with high speed.

\subsection{The Self-organizing Electromagnetic Field Criticality and the Creation of the Spatial Super-Compression of Continuous Media}

In these experiments the unhomogeneity distribution of gas media densities is demonstrated during supplying of highfrequency electro-magnetic field, antenna of HF field is by 20 $\mathrm{cm}$ up from edge of the cone of dynamic emitter which is made from steel. At our early works we discussed that the jet emites and the area is glowing. (Fig. 15) [13]. At Fig. 15 we observe super-compression at the metal surface which leads to glowing of air at infrared area (below part of the picture) and at the violet and ultraviolet and area of spectrum (at the dynamic emitter cone top). At Fig. 16 an air jet are outflowing which supplies from the chamber under pressure $2 * 10^{5} \mathrm{~Pa}$ [13], [15]. At Fig. 16 the pressure is $2 * 10^{5} \mathrm{~Pa}$, there is no any pressure and a jet at the Fig. 17 and Fig. 18. The field is $1.5 \mathrm{MHz}$ at the Fig. 17 and it is less than $1 \mathrm{MHz}$ at the Fig. 18. The formation of the high air densities occurs at the edge of the nozzle and antenna is up from it. The situation when the pressure is strongly decreased and the field is also decreased to order is shown on the Fig. 16, the field doesn't change at the Fig. 17, there no any pressure. There is no any pressure and the jet at 
the Fig. 18, we just increased the magnitude of the field to less than $1 \mathrm{MHz}$, and power of the source supplying the field is 1 $\mathrm{kW}$. White arrow show on the formation of high media and electromagnetic energy density area.

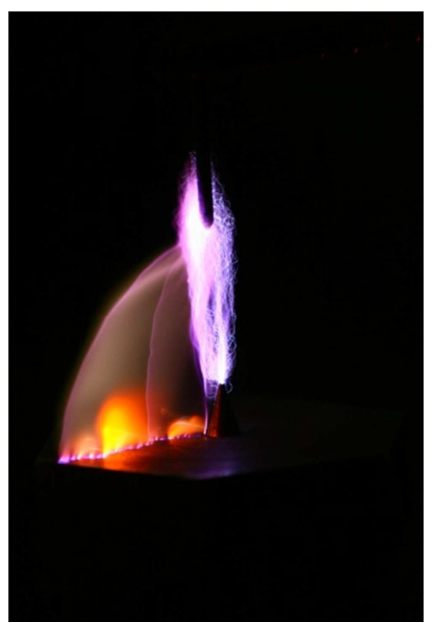

Fig. 15. The formation of red formation of high gas densities on the nozzle surface. The pressure is $4 * 10^{5} \mathrm{~Pa}$.

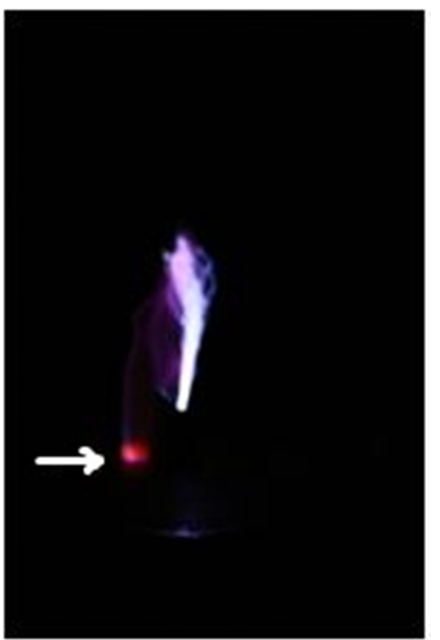

Fig. 16. The same process under pressure $2 * 10^{5} \mathrm{~Pa}$. Red formation is not far from nozzle cut on the cone surface.

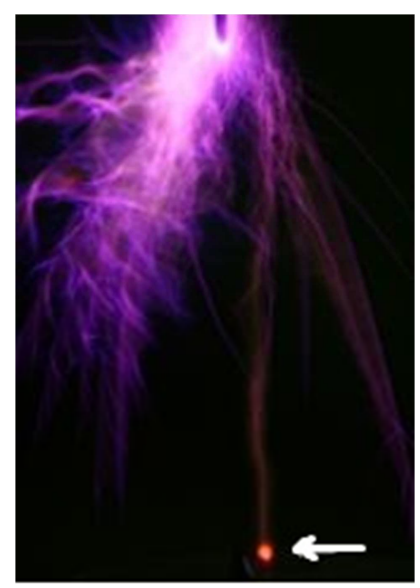

Fig. 17. The formation of high gas densities on the edge of the nozzle. The $1.5 \mathrm{MHz}$ field is supplied on the antenna. There is no any jet.

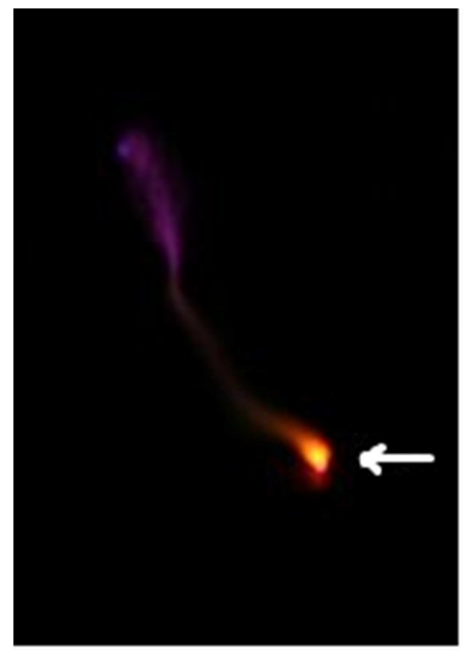

Fig. 18. There is no any pressure and the jet on this photo. We see clearly the formed high gas density and the field which is supplied on antenna is less than $1 \mathrm{MHz}$.

\section{Discussion of Experimental Results}

The obtained results of the experiments attest about mechanism of the spatial super-compression and the glowing of the continuous media based on that the strong compression energy transforms in electromagnetic radiation energy.

At the first experiment we observe the formation of spherical symmetrical high gas densities in the supersonic jet structure. We early have discussed that during outflowing of air the spiral structure occurs where super-compression and its glowing appear. This is a result of transformation of $\mathrm{HF}$ sound energy to HF electromagnetic field energy. Under the adiabatic conditions of gas outflowing the transformation of HF fields as acoustic ones as electromagnetic ones lead to the spatial super-compression of continuous media. So we presented two types of experimental results in which at the one case the spatial super-compression of continuous media happens without using of $\mathrm{HF}$ electromagnetic fields and at the other case with using of HF electromagnetic fields in which the spatial super-compression and the glowing are observed. At the second case we can visualize this phenomenon and register the spectrum. We presented these data at the previously works. At the next experiments we studied the interaction supersonic jet with thermal flow and obtained results attest about the spatial super-compression of continuous media. Using the dynamic emitter we observed these phenomena as during the jet as without it.

Total result of the presented experimental work and our calculations is the formation of unhomogeneity gas density distribution and instability which lead to the self-organizing criticality of HF electromagnetic field and these factors are cause of the spatial super-compression of continuous media. However the HF electromagnetic and sound fields only help us to visualize this phenomenon and register spectral characteristics and also make energy estimates of the glowing, but the main mechanism is the transformation of the spatial super-compression of the continuous media energy into the light energy. 


\section{Conclusions}

1. Dynamic emitter is unique construction due to we observe the spatial super-compression of continuous media which are caused by unhomogeneity gas density distribution and instability as without using HF electromagnetic fields as with using HF fields.

2. The formation of the spatial super-compression of continuous media and the glowing is caused by the transformation of the spatial strong compression of the continuous media energy into the high density light energy.

3. All these experiments on the spatial super-compression of continuous media and the formation of high density light energy in small volumes became total result of published works. We think that obtained results can become a good laboratory model for the researching of this unique phenomena related to energetic area of science.

\section{Acknowledgements}

Author is deeply grateful to A. I. Klimov from United Institute of High Temperature of Russian Academy of Sciences for their providing of visualization experiments and grateful to K. E. Markovets from M. V. Lomonosov MSU Physical Department, A. E. Yunovich from M. V. Lomonosov Moscow State University Semiconductor Physics Department for kindly provided equipment for spectral measurements.

\section{References}

[1] Kh. Khasanov, "Visualization of Super-Compressibility in Supersonic Spiral-Twisted Jets", Physics Letters A, Vol. 376, No. 5, 2012, pp. 748-753.
[2] Kh. Khasanov, "Emission of High Energy during SuperCompressibility of Supersonic Jets", Open Journal of Fluid Dynamics, 2012, 2, 172-179, pp. 174-175.

[3] Lukyanov S. Y. "Hot plasma and controlled nuclear synthesis". - M: Science, 1975, p. 359.

[4] Van Marum M 1790 Proc. 4th Int. Conf. on Dense Z-Pinches, Am. Inst. Phys. Woodbury, New York, 1997i.

[5] Pollock J A and Barraclough S, 1905 Proc. R. Soc. New South Wales 39, p. 131.

[6] Artsimovich A. A, "Controlled thermonuclear reactions". M: "Physmathspub", 1961, p. 347.

[7] Vladimirov V. V. // Successes of physical sciences. 1975, V. 117, №1, p. 79.

[8] W.H. Bennett // Phys. Rev. 1934, v. 45, p. 890.

[9] P.H. Robert // Astrophys. J. 1956, v. 124, p. 430.

[10] Kh. Khasanov, "Double Spiraled Supersonic Jet," Fluid Dynamics, Vol. 46, No. 3, 2011, pp. 433-436.

[11] Kh. Khasanov and S. V. Petukhov, "Dynamic Emitter," PF Patent No. 2058196, 1996.

[12] Kh. Khasanov, "Visualization of super-compressibility in supersonic spiral-twisted jets", Physics Letters A, 376 (2012) 748-752, p. 751.

[13] Kh. Khasanov, "Super-Compressibility Phenomenon", Journal of Modern Physics, 2013, 4.

[14] Kh. Khasanov, "Electromagnetic Super-Compressibility", Material Science \& Engineering, J Material Sci Eng Volume 2, Issue 4, 1000131, ISSN: 2169-0022 JME, 2013, pp. 3-4. http://dx.doi.org/10.4172/2169-0022.1000131.

[15] Kh. Khasanov, "Visualization of Bi-Spiral Twisted Supersonic Gas Flow," The 8th Pacific Symposium of Flow Visualization and Image Processing, Moscow, 2011, p. 51. 